\title{
Next-Generation Therapeutic Concepts for Atherosclerosis: Focus on Cell Specificity and Noncoding RNAs
}

\author{
Lucia Natarelli ${ }^{1}$ Christian Weber ${ }^{1,2,3}$ \\ ${ }^{1}$ Institute for Cardiovascular Prevention, Ludwig-Maximilians- \\ Universität (LMU) München, Munich, Germany \\ ${ }^{2}$ German Center for Cardiovascular Research (DZHK), Partner Site \\ Munich Heart Alliance, Munich, Germany \\ ${ }^{3}$ Cardiovascular Research Institute Maastricht, University of \\ Maastricht, ER Maastricht, the Netherlands
}

Thromb Haemost 2019;119:1199-1201.

\begin{abstract}
Address for correspondence Christian Weber, MD, Institute for Cardiovascular Prevention, LMU Munich, Pettenkoferstraße 9, 80336 Munich, Germany (e-mail: chweber@med.Imu.de).
\end{abstract}

an antibody to interleukin (IL)-1 $\beta$, or other modalities targeting IL-6 or IL-1 receptors are aimed at more specifically reducing inflammatory activity during atherosclerosis. ${ }^{1}$ However, current anti-inflammatory and antithrombotic therapies are far from lacking side effects, although the risk-benefit ratio still makes them an indispensable choice improving the life expectancy of patients with cardiovascular diseases.

The exponential growth of genome-wide association studies (GWAS), single-cell sequencing databases, and context-based text mining has brought to light the existence of complex regulatory networks triggering cardiovascular disease. Among these, the heterogeneity and interconnection of vascular cell populations, as well as their selective contribution to distinct stages of atherosclerosis particularly stand out (-Fig. 1). Moreover, GWAS have shown disease-linked genetic variation in the nonprotein-coding sequence space, which are actively transcribed to noncoding ribonucleic acids (ncRNAs), that is, micro- and long ncRNAs (miRNAs and IncRNAs). This novel class of ncRNAs is differentially expressed in diseased tissues and act as epigenetic modulators of gene expression. Indeed, the Part 2 of the Theme Issue on atherosclerosis and atherothrombosis brings together the innovative and provocative view of expert scientists on novel therapeutic perspectives. Among these, the identification of phenotype- and cell-to-cell interactionrelated molecules, as well as epigenetic modulatory-related molecules, as novel and selective therapeutic targets to treat atherosclerosis.

Review articles by Busygina et $\mathrm{al}^{2}$ and Pircher et $\mathrm{al}^{3}$ underscore a fundamental problem with current immunotherapies. Indeed, antiplatelet and collagen inhibitors, such as the first-inclass oral irreversible Bruton tyrosine kinase (Btk) inhibitors

(c) 2019 Georg Thieme Verlag KG Stuttgart · New York
DOI https://doi.org/ 10.1055/s-0039-1693412. ISSN 0340-6245. received

June 18, 2019

accepted

June 18, 2019 


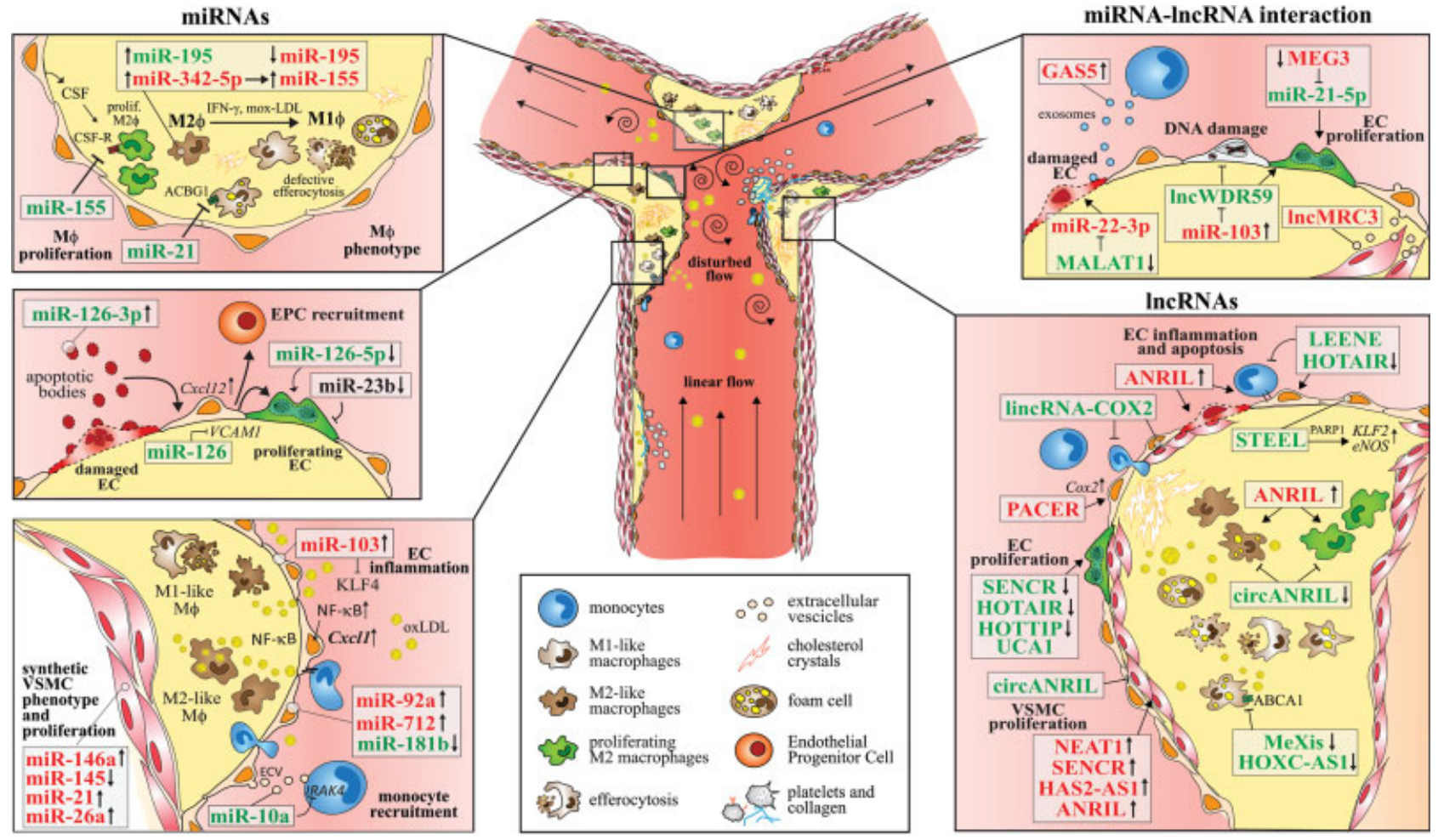

Fig. 1 Micro (miRNAs)- and long noncoding ribonucleic acids (IncRNAs') role during atherosclerosis. Graphical overview of the major miRNAs and IncRNAs reviewed in the Theme Issue. MiRNAs and IncRNAs contribute to atherosclerosis alone or by affecting their reciprocal activation. Hyperlipidemia impairs endothelial regeneration inhibiting miR-126-5p expression and promoting proinflammatory miR-92a and miR-103. The majority of IncRNAs are downregulated during atherosclerosis, suggesting that their overexpression might be promising to prevent plaque progression. Indeed, MALAT1, HOTAIR, IncWDR59, and circANRIL sustain endothelial regeneration and prevent their apoptosis and inflammation. However, linear ANRIL, IncMRC3, and PACER promote endothelial inflammation and smooth muscle cell (SMC) cup formation. Monocyte recruitment is enhanced by endothelial release of miR-10a. At early stages of atherosclerosis, miR-155 and miR-195 inhibit M2-like macrophage proliferation. However, miR-342-5p-mediated increase of miR-155 expression, miR-21 overexpression, and miR-195 inhibition, all together sustain M1-like inflammatory macrophage polarization and defective efferocytosis. CircANRIL, MeXis, and IncRNA HOCX-AS1 are downregulated during these stages, and therefore cannot inhibit chronic inflammation. SMC miRNAs and IncRNAs, such as miR-146a/145/21/26a, NEAT1, SENCR, and ANRIL, seem to synergistically promote their proliferation and to contribute at cup formation. CircANRIL is instead acting as SMC proliferative agonist.

(ibrutinib and acalabrutinib), show bleeding side effects. These are partly ascribable to complex off-target mechanisms of drug concentrations used to treat, for example, B cell malignancies and not required for the inhibition of glycoprotein VI-mediated response of platelets to low collagen or plaque. Busygina et $\mathrm{al}^{2}$ summarized the last clinical trials on antithrombotic drugs and underlie the relevance of a short-term application of novel reversible Btk inhibitors to avoid bleeding side effects. At the same time, Pircher et $\mathrm{al}^{3}$ introduce novel therapeutic targets with focus on cell-to-cell interaction. Following plaque rupture, highly thrombogenic plaque content become exposed to blood stream and mediate platelet and neutrophil recruitment. Neutrophils and platelets reciprocally sustain their activation and promote inflammation by exposing adhesion molecules, such as P-selectin, P-selectin glycoprotein ligand 1, and, as recently emerged, by releasing citrullinated histone 3-rich extracellular traps (neutrophil extracellular traps [NETs]). Notably, neutrophil-deriving NETs can serve as scaffold for platelet aggregation, and sustain inflammation and thrombosis. Hence, Pircher et $\mathrm{al}^{3}$ introduce novel short-term platelet inhibition treatments in acute thrombosis aimed to inhibit neutrophil-platelet aggregation, with potentially less side effects than those from established long antithrombotic therapies.

Monocyte-derived macrophages are a perfect example of stage-dependent cell phenotypic change during atherosclerosis. Aside the concept that macrophage polarization can be reprogrammed by inflammatory stimuli, Stremmel et $\mathrm{al}^{4}$ here underline how the shift between inflammatory (M1-like) and proliferative (M2-like) macrophages is the resultant of their metabolic changes. Notably, M1-like macrophages utilize glycolytic metabolism while M2-like macrophages feature mitochondrial oxidative phosphorylation and fatty acid metabolism. However, macrophages metabolism is altered during atherosclerosis. Herein, Stremmel et al interestingly discuss how immunometabolism might be used to target selective macrophage subpopulation in dependence of their form of energy supply.

Heterogeneity of cells involved in atherosclerosis is therefore a critical point to consider to generate more selective therapeutic drugs and to reduce side effects. Moreover, it could also explain why current therapies do not show the same beneficial effects in all patients. The epigenetic aspect could be the missing link to develop more selective and effective therapies. The review articles by Eckardt et $\mathrm{al}^{5}$ Paloschi et al, ${ }^{6}$ and Holdt 
et $\mathrm{al}^{7}$ exactly describe glycan-binding proteins, miRNAs, and IncRNAs selective epigenetic biomarkers as novel and potential therapeutic targets to treat atherosclerosis. Vascular glycans are dynamically influenced by the physiological state of the cells, which is read and translated into function by glycan-binding proteins. Therefore, glycans represent the "footprint" of the cells at a selective pathophysiological stage. Eckardt at $\mathrm{al}^{5}$ discuss how glycans could be used as intermediate between a drug and its target to increase drug specificity. Notably, mass spectrometry and nuclear magnetic resonance studies identified lectins and galectins as therapeutic biomarkers and thrombotic targets. However, the complexity of glycan structures makes their identification and structural function analysis difficult, and at the moment only heparins have been customized for anti-inflammatory therapies.

ncRNAs are a new class of - "mechanistically easy-tostudy" - epigenetically modulated molecular markers that offer a selective therapeutic advantage. Paloschi et $\mathrm{al}^{6}$ and Holdt et al ${ }^{7}$ describe selective inhibition/overexpression of miRNAs and lncRNAs as more off-target, easy delivery, and less side effect therapeutic alternative to conventional immunotherapies. In particular, Paloschi et al overview the role of ncRNAs in myeloid cells, or released from arterial cells for myeloid recruitment, to modulate inflammatory cascade during vascular disease. Notably, administration of miR126-5p mimics restore impaired endothelial proliferation, whereas miR-181b and miR-92a inhibition reduces inflammation. Similarly, exosome-released lncRNCR3 protect against hypercholesterolemia-induced EC and SMC dysfunction, whereas lincRNA-p21, MeXis, and MALAT1 promote macrophage anti-inflammatory role and cholesterol efflux. As underlined in the review by Holdt et al, ${ }^{7}$ current RNA therapeutic trials exclusively involve small interfering RNAs and mRNAs, but not yet lncRNAs. However, to consider IncRNA-centered therapies we should first take some issues into consideration. First, some lncRNAs are poorly conserved between mouse and human. Second, some of the ncRNAs reported as potential therapeutic candidates show different effects depending on the disease considered. For example, MALAT1 overexpression reduces atherosclerosis, but it is also cancer-promoting. Third, miRNAs and IncRNAs can affect their activation, playing opposite roles during atherosclerosis. Indeed, sponge activity of IncRNA MEG3 and MALAT1 on miR-21-3p and miR-22-3p, respectively, affect EC proliferation and apoptosis during atherosclerosis. Moreover, one miRNA can target bot mRNAs and lncRNAs, such as atheromiR-103, which promote EC inflammation by targeting $\mathrm{KLF} 4,{ }^{8}$ and impairs EC regeneration by targeting IncWDR59. ${ }^{9}$ To avoid side effects and off-targeting, Holdt et al $^{7}$ describe the use of synthetic circular IncRNAs, which can play even a different role compared with their endogenous counterparts, that can be packaged in lipid vesicles, conjugated to antibodies, or (current modern approach) eluted from coated stents and perivascular hydrogels. Accordingly, while linear ANRIL promote atherosclerosis, circular ANRIL mediates opposite, protective effects.

Reading these reviews reveals how innovative but at the same time how complex it will be to consider epigenetically relevant molecules as more selective alternative therapies. The context-based atheMir database introduced by Joppich et al ${ }^{10}$ is a database of miRNA-gene interaction networks that offer a good starting point to investigate epigenetic molecule interconnections in a cell-to-cell and phase-context dependent manner during atherosclerosis. Based on scientifically supported evidences and reported interactions, atheMir explores new selective miRNA-gene interaction hypothesis in cardiovascular diseases, that is, endothelial and monocytes miR-126 and miR-155/222 role in early atherosclerotic stages, and SMC miR-98 and miR-504 role at later stages.

Although considered the "dark side of the genome," this ncRNA force in humans probably derives from the ability (or attempt) of nature being to develop a new evolutionary stage to adapt more easily to the cumulatively higher risk of cardiovascular diseases related to a longer life time.

\section{Conflict of Interest}

None declared.

\section{References}

1 Lutgens E, Atzler D, Döring Y, Duchene J, Steffens S, Weber C. Immunotherapy for cardiovascular disease. Eur Heart J 2019. Doi: 10.1093/eurheartj/ehz283

2 Busygina K, Denzinger V, Bernlochner I, Weber C, Lorenz R, Siess W. Btk inhibitors as first oral atherothrombosis-selective antiplatelet drugs? Thromb Haemost 2019;119(08):1212-1221

3 Pircher J, Engelmann B, Massberg S, Schulz C. Platelet-neutrophil crosstalk in atherothrombosis. Thromb Haemost 2019;119(08): 1274-1282

4 Stremmel C, Stark K, Schulz C. Heterogeneity of macrophages in atherosclerosis. Thromb Haemost 2019;119(08):1237-1246

5 Eckardt V, Weber C, von Hundelshausen P. Glycans and glycanbinding proteins in atherosclerosis. Thromb Haemost 2019;119 (08):1265-1273

6 Paloschi V, Winter H, Viola J, Soehnlein O, Maegdefessel L. Mechanistic links between non-coding RNAs and myeloid cell inflammation in atherosclerosis. Thromb Haemost 2019;119(08): 1205-1211

7 Holdt LM, Kohlmaier A, Teupser D. Long non-coding RNAs of the arterial wall as therapeutic agents and targets in atherosclerosis. Thromb Haemost 2019;119(08):1222-1236

8 Hartmann P, Zhou Z, Natarelli L, et al. Endothelial Dicer promotes atherosclerosis and vascular inflammation by miRNA-103-mediated suppression of KLF4. Nat Commun 2016;7:10521

9 Natarelli L, Geißler C, Csaba G, et al. miR-103 promotes endothelial maladaptation by targeting lncWDR59. Nat Commun 2018;9 (01):2645

10 Joppich M, Weber C, Zimmer R. Using context-sensitive text mining to identify miRNAs in different stages of Atherosclerosis. Thromb Haemost 2019;119(08):1247-1266 